\title{
Exactitud de pruebas clinicas para verificar la adecuada colocación del catéter venoso central en Urgencias.
}

\author{
Jorge Ayón-Aguilar ${ }^{1}$, Norberto Martínez-Luna ${ }^{2}$, Fernando Fernández-Lucas ${ }^{2}$, \\ Socorro Méndes-Martíne: ${ }^{3}$ y Patricia Seefoó-Jarquín ${ }^{4}$ \\ ${ }^{1}$ Coordinación Auxiliar Médica de Investigación en Salud, Instituto Mexicano \\ del Seguro Social, Puebla, México. \\ ${ }^{2}$ Servicio de Urǵencias de Adultos. Hospital General Regiional número 36, Puebla, \\ Instituto Mexicano del Seguro Social. Puebla, México. \\ ${ }^{3}$ Coordinación de Planeación y Enlace Institucional, Instituto Mexicano del Seguro \\ Social. Puebla, México. \\ ${ }^{4}$ Coordinación Clínica de Medicina Interna, Hospital General de Zona número 1, \\ Instituto Mexicano del Seguro Social. Tlaxeala, México.
}

Palabras clave: catéter venoso central; radiografía de tórax; ultrasonido.

Resumen. La utilidad del catéter venoso central (CVC) depende de su adecuada colocación y la radiografía de tórax es el estándar de oro para corroborarla. Para este fin se utilizan pruebas clínicas. El objetivo del presente estudio fue determinar la sensibilidad, especificidad y valores predictivos de cinco pruebas clínicas, para verificar la adecuada colocación del CVC, por lo que se realizó un estudio en 128 pacientes ingresados al servicio de Urgencias, con necesidad de colocación de CVC; se realizaron cinco pruebas clínicas: determinación de arritmias (Ar), retorno venoso (RV), oscilación de presión venosa central (OPVC), medición externa (ME) y gasometría venosa central (GVC); la posición del catéter se corroboró con radiografía de tórax. El 97\% presentó adecuada colocación del CVC en la radiografía de tórax; los resultados de sensibilidad (Se), especificidad (Sp), valores predictivos positivo (VPP) y negativo (VPN) de las pruebas fueron: Ar: 59,6\%, 50\%, 97,3\% y 3,8\%; RV: 100\%, 0\%, 96,8\% y 0\%; OPVC: 99,1\%, 25\%, 97,6\% у 50\%; ME: 100\%, 0\%, 96,8\% у 0\%; GVC: 96,7\%, 0\%, 96,7\% у 0\%, respectivamente. Concluimos que si las pruebas clínicas OPVC, Ar y RV resultan positivas, por sus altos valores de sensibilidad y valor predictivo positivo, podrían sustituir la necesidad de realizar una radiografía de tórax inmediata y no retrasar el uso del CVC, siempre y cuando no se sospechen complicaciones secundarias tempranas a la colocación del mismo.

Autor de correspondencia: Jorge Ayón-Aguilar, Coordinación Auxiliar Médica de Investigación en Salud, Delegación Puebla, Instituto Mexicano del Seguro Social, 4 norte 2005, Colonia Centro, C.P. 72000, Puebla, Puebla, México. Teléfono: 2228-61-89-44. Correo electrónico: jorgeayonaguilar@gmail.com 


\title{
Accuracy of clinical tests to verify the proper placement of central venous catheter in the Emergency Room.
}

\author{
Invest Clin 2021; 62 (3): 219-229
}

Key words: central venous catheter; chest X-ray; ultrasound.

\begin{abstract}
The utility of the central venous eatheter (CVC) depends on its proper placement; chest X-ray is the gold standard for corroborating it. We studied five clinical tests as useful tools for determining the CVC placement. The objective of the present study was to determine the sensitivity, specificity and predictive values from five clinical tests to verify the proper CVC placement, in patients admitted to the ER in need of a CVC procedure. Five clinical tests were performed: determination of arrhythmias (Ar), venous return (VR), central venous pressure oscillation (CVPO), external measurement (EM) and central venous blood gases (CVBG); the position of the catheter was corroborated with chest X-ray. Of the 128 patients who were included, 97\% had adequate placement of the GVC on chest X-ray. The results of sensitivity (Se), specificity ( $\mathrm{Sp}$ ), positive predictive value (PPV) and negative predictive value (NPV) of the tests were: Ar: 59.6\%, 50\%, 93.7\% and 3.8\%; VR: 100\%, 0\%, 96.8\% and $0 \%$; CVPO: $99.1 \%, 25 \%, 97.6 \%$ and 50\%; EM: $100 \%, 0 \%, 96.8 \%$ and $0 \%$; CVBG: $96.7 \%$, 0\%, 96.7\% y 0\%, respectively. We conclude that due to their high sensitivity and positive predictive values, if the CVPO, Ar and VR clinical tests are positive, they could replace the need for an immediate chest X-ray and not delay the use of the CVC, as long as no early complications in its placement are suspected.
\end{abstract}

Recibido: 08-09-2020Ａceptado: 07-05-2021

\section{INTRODUCCIÓN}

Los catéteres venosos centrales (CVC), se utilizan ampliamente en pacientes críticamente enfermos; su colocación y permanencia están asociadas a complicaciones como malposición, hidro-neumotórax, arritmias, taponamiento cardíaco, embolismo aéreo, punción de grandes vasos, lesión del plexo braquial/nervio frénico y trombosis venosa, que se han reportado en un rango de 0,4 a $20 \%$ en diferentes estudios, dependiendo de la experiencia del operador (1-4). Para determinar la adecuada colocación del CVC, la punta del mismo debe encontrarse en el tercio proximal de la vena cava superior, en la aurícula derecha o en la vena cava inferior (5-7). El es- tándar de oro para verificar su posición sigue siendo la radiografía de tórax $(8,9)$.

En los últimos 20 años, se ha descrito la utilización de diferentes métodos para verificar la adecuada colocación del CVC, tales como electrocardiografía, fluoroscopía, manometría y ultrasonografía (10-17), sin embargo, los equipos necesarios para estos estudios, no se encuentran disponibles en todas las áreas de Urgencias, en países con economías emergentes; en este contexto, algunas pruebas clínicas podrían ser útiles para verificar la adecuada colocación del GVC, tales como determinación de arritmias (Ar) (18), retorno venoso (RV), oscilación de la presión venosa central (OPVC), medición externa de la longitud del catéter (ME) e in- 
cluimos la gasometría venosa central (GVC), como otra opción (19-23).

En la actualidad, no hay muchos estudios que determinen la sensibilidad y especificidad de estas cinco pruebas clínicas, como herramientas útiles para incluirlas en la práctica clínica diaria como auxiliares en la verificación de la adecuada colocación del CVC, mientras se espera la realización de la radiografía de tórax. Con base en ello, este estudio tiene la finalidad de determinar la sensibilidad, la especificidad y los valores predictivos de las cinco pruebas clínicas que proponemos, al compararlas con el estándar de oro: la radiografía de tórax.

\section{Pacientes y Métodos}

El presente estudio fue transversal, prueba versus prueba, se comparó cada una de las pruebas por separado, con la radio- grafía de tórax de cada paciente, y se realizaron las pruebas consecutivas. Realizado entre septiembre 2017 y agosto 2018, en el Hospital General Reǵional número 36, IMSS, Puebla, México (Fiǵ. 1).

Nos apegamos a Normas Éticas: declaración de Helsinki de la Asociación Médica Mundial; Reglamento de la Ley General de Salud en Materia de Investigación para la Salud en México; Código de Nuremberǵ e Informe Belmont. El estudio fue autorizado por los Comités Locales de Ética e Investigación con número de Registro Nacional R-2017-210254. El reclutamiento de los pacientes fue consecutivo; una vez cumplidos los criterios de selección, se les explicó de manera clara y detallada, el procedimiento al que serían sometidos, sus riesgos y tratamientos alternativos. El paciente o su familiar responsable firmaron el Consentimiento Informado.

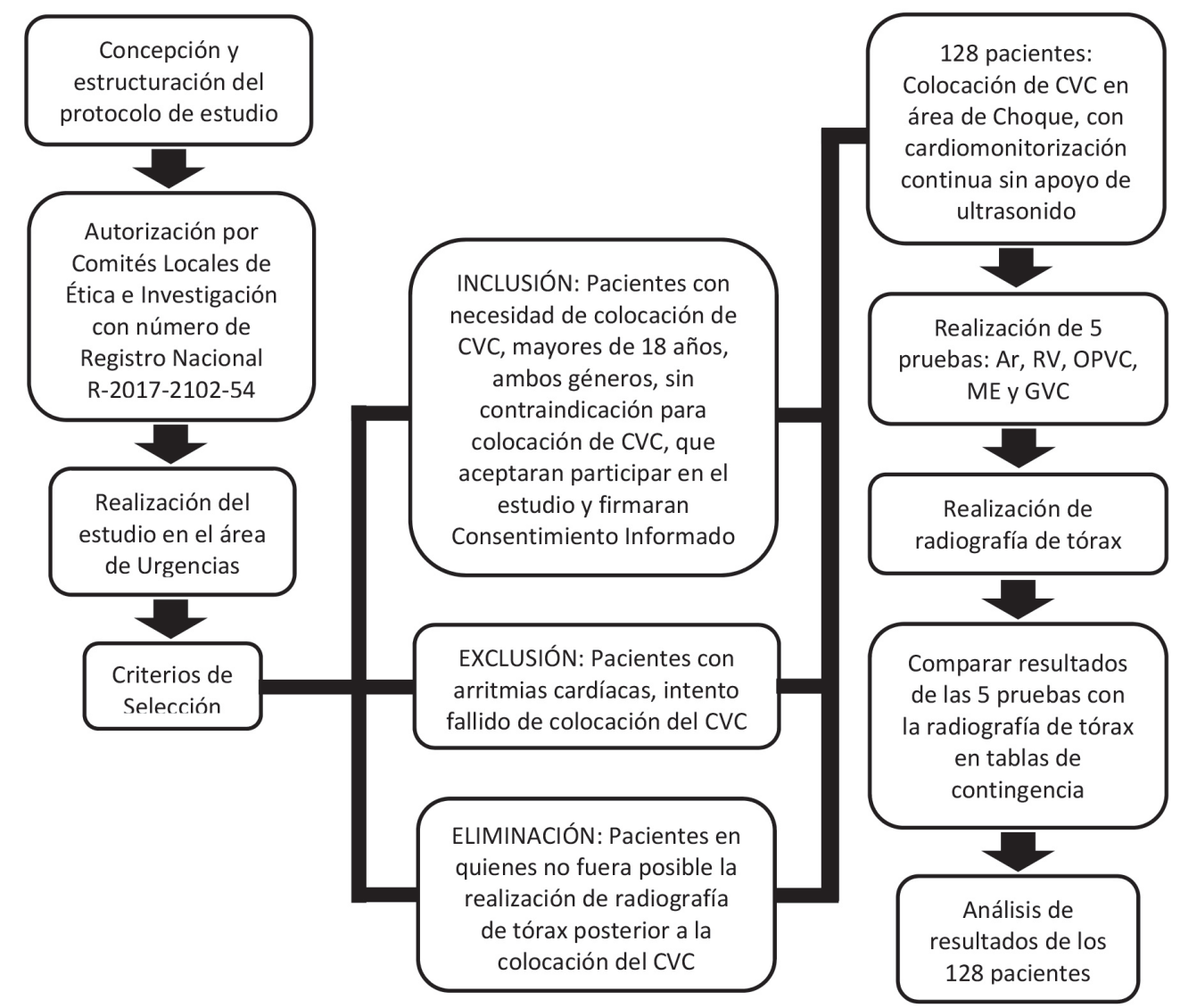

Fig. 1 . 
La colocación del CVC, marca Arrow, calibre 7 Fr doble lumen, fue realizada por un solo médico urgenciólogo, únicamente por vía subclavia derecha, en área de Choque, con cardiomonitorización continua, sin apoyo de ultrasonido durante el procedimiento; ya colocado el CVC, el mismo médico urǵenciólogo realizó las cinco pruebas clínicas en los pacientes, para verificar la adecuada colocación del CVC: Ar (alteraciones del ritmo cardíaco registradas en el monitor, causadas por el contacto de la punta del CVC con el endocardio), RV (obtención de sangre mediante la aspiración con jeringa a través del CVC y/o colocar la bolsa de infusión por debajo del nivel del corazón del paciente), OPVC (oscilación de columna sanguínea en el lumen del GVC durante los movimientos de ventilación del paciente), ME del CVC (medición en centímetros de la longitud del catéter desde el sitio de inserción hasta la zona más distal del mismo, previa y posterior a su colocación, la cual debe ser mayor a 2,5 cm) y GVC (análisis de gases en la sangre obtenida del CVC). Valores para presión de oxígeno $\left(\mathrm{PaO}_{2}\right)$ y saturación de oxígeno $\left(\mathrm{SatO}_{2}\right)$ venosos aceptados: $30-50 \mathrm{mmHg}$ y menor a $75 \%$, respectivamente. Posteriormente, a todos los pacientes se les realizó radiogra- fía de tórax (imaǵen radiográfica que debe mostrar la punta del CVC a la altura de la carina o aurícula derecha y ausencia de hidro o neumotórax. Estos hallazgos afirmarían que el GVC estaría en adecuada colocación). La positividad o negatividad de las pruebas fue determinada por el mismo médico urgenciólogo que colocó el CVC. Un total de 128 pacientes fueron incluidos en el estudio, 53\% mujeres $(n=68)$ y $47 \%$ hombres $(n=60)$; su distribución por grupo de edad se presenta en la Fig. 2.

Métodos estadísticos: Para determinar Sensibilidad (Se), Especificidad (Sp), Valor Predictivo Positivo (VPP) y Valor Predictivo Negativo (VPN) con intervalos de confianza al 95\% de las variables, se tomó como estándar de oro la radiografía de tórax. Se utilizaron tablas de contingencia de $2 \mathrm{x} 2$ y Curvas ROC con su valor de Área Bajo la Curva (ABC), enfrentando cada una de las pruebas clínicas por separado, con la radiografía de tórax. Se utilizó el programa estadístico SPSS v. 22.

\section{RESULTADOS}

Los valores absolutos y porcentuales obtenidos de las cinco pruebas clínicas por

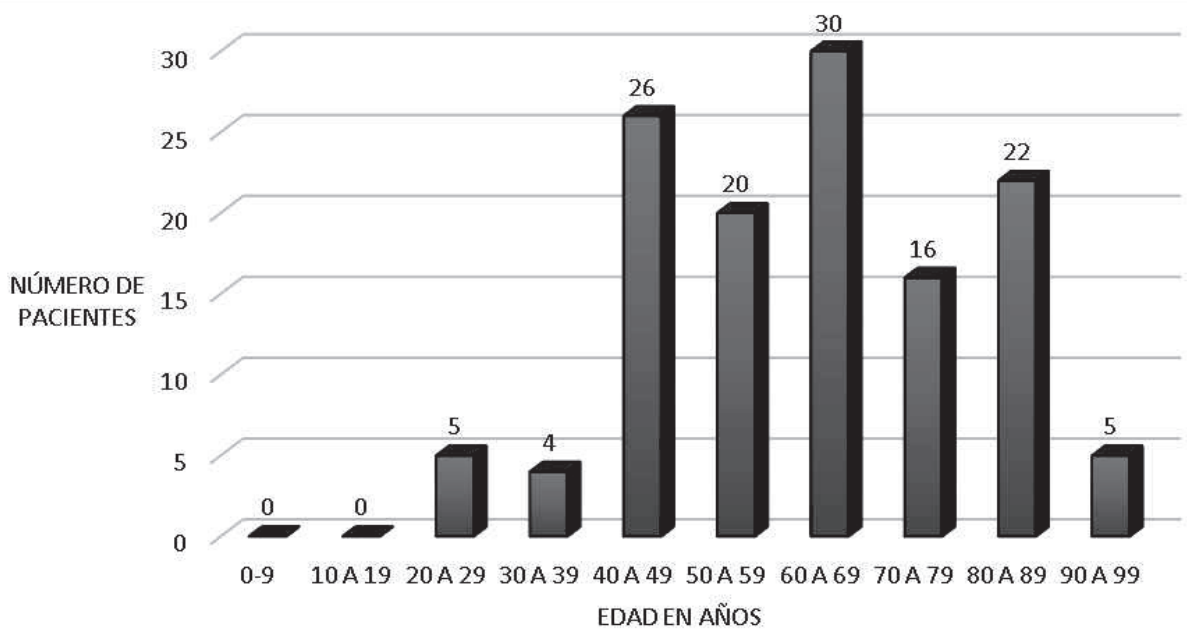

Fig. 2. Distribución por ǵrupos de edad de los pacientes incluidos en el estudio de pruebas clínicas para verificar la adecuada colocación del catéter venoso central en Urǵencias. 
separado, en pruebas consecutivas, al realizar tablas de contingencia de $2 \times 2$ y compararlas con la radiografía de tórax se muestran en las Tablas I, II, III, IV y V. Las curvas ROC para demostrar la Se y Sp, así como sus valores del $\mathrm{ABC}$ de las pruebas, se muestran en la Fig. 3. Los resultados globales de la exactitud de las cinco pruebas, se muestran en la Tabla VI, donde se demuestra que son altamente sensibles y con un VPP elevado, resaltando de entre las cinco pruebas, la OPVC, que obtuvo los mejores valores de Se, Sp, VP y ABC.

La única complicación que se registró durante el estudio, secundaria a la colocación del CVC, fue la presencia de hematoma en el sitio de la punción, presentándose en 3,1\% (4 pacientes). El tratamiento para esta complicación fue inmediato por medio de compresión del sitio, resolviéndose en todos los casos.

\section{DISCUSIÓN}

En la actualidad, la utilización de aceesos venosos centrales en los pacientes críticos atendidos en las áreas de Urgencias, es fundamental y se recomienda su uso, cuando los beneficios superan a los riesgos, ya que en últimos años se ha preferido practicar terapéutica mínimamente invasiva. Recientemente, se ha optado por la ultrasonografía tanto como apoyo durante la colocación de los CVC, como para verificar la adecuada colocación de las líneas centrales $(2,12)$. Chebl y col., en un metanálisis realizado en el 2017, reportaron que el ultrasonido mejorado con contraste, tiene una Se, Sp, VPP y VPN de 72, 100, 92 y 98,5\%, respectivamente (25), e incluso, hay autores que han reportado que la ultrasonografía puede sustituir en alguunos casos a la radiografía de tórax $(9,15)$, con disminución de los riesgóos y tiempos del procedimiento, costos monetarios, traslado de equipo o paciente para la toma de radiografía de control, requerimiento de personal y morbilidad secundaria a la exposición radiante.

Ubicados en la situación de economías emergentes como la de México, no se cuenta con equipo de ultrasonido en todos los Servicios de Urgencias y, en caso contrario, no está disponible las 24 horas del día; asimismo, en la mayoría de los casos, no se tiene la

TABLA I

VALIDEZ DE ARRITMIA CARDÍACA VS RADIOGRAFÍA DE TÓRAX PARA VERIFICAR LA ADECUADA COLOCACIÓN DEL CATÉTER VENOSO CENTRAL EN URGENCIAS.

\begin{tabular}{|c|c|c|c|c|}
\hline & & Radio & tórax & \\
\hline & & $\begin{array}{c}\text { Positivo } \\
\text { N (\%) }\end{array}$ & $\begin{array}{c}\text { Negativo } \\
\text { N (\%) }\end{array}$ & $\begin{array}{l}\text { Total } \\
\text { N (\%) }\end{array}$ \\
\hline & Positivo & $74(59,6)$ & $2(50)$ & $76(59,3)$ \\
\hline Ar & Negativo & $50(40,3)$ & $2(50)$ & $52(40,6)$ \\
\hline & Total & 124 & 4 & 128 \\
\hline & & & & \\
\hline & & LI & LS & \\
\hline Se & $59,68 \%$ & 50,47 & 68,27 & \\
\hline Sp & $50 \%$ & 9,19 & 90,81 & \\
\hline VPP & $97,37 \%$ & 89,95 & 99,54 & \\
\hline VPN & $3,85 \%$ & 0,67 & 14,33 & \\
\hline
\end{tabular}

Vol. 62(3): $219-229,2021$ 


\section{TABLA II}

VALIDEZ DE RETORNO VENOSO VS RADIOGRAFÍA DE TÓRAX PARA VERIFICAR LA ADECUADA COLOCACIÓN DEL CATÉTER VENOSO CENTRAL EN URGENCIAS.

\begin{tabular}{|c|c|c|c|c|}
\hline & \multicolumn{4}{|c|}{ Radiografía de tórax } \\
\hline & & $\begin{array}{c}\text { Positivo } \\
\text { N (\%) }\end{array}$ & $\begin{array}{c}\text { Negativo } \\
\text { N (\%) }\end{array}$ & $\begin{array}{l}\text { Total } \\
\text { N (\%) }\end{array}$ \\
\hline \multirow{5}{*}{ RV } & Positivo & $124(100)$ & $4(100)$ & $128(100)$ \\
\hline & Negativo & 0 & 0 & 0 \\
\hline & Total & 124 & 4 & 128 \\
\hline & \multicolumn{4}{|c|}{ IC 95\% } \\
\hline & & LI & LS & \\
\hline $\mathrm{Se}$ & $100 \%$ & 96,25 & 99,93 & \\
\hline Sp & $0 \%$ & 2,35 & 60,42 & \\
\hline VPP & $96,88 \%$ & 91,71 & 98,99 & \\
\hline
\end{tabular}

" RV: Retorno Venoso; Se: Sensibilidad; Sp: Especificidad; VPP: Valor Predictivo Positivo; IC 95\%: Intervalo de Confianza al 95\%; LI: Límite Inferior; LS: Límite Superior.

\section{TABLA III}

VALIDEZ DE OSCILACIÓN DE LA PRESIÓN VENOSA CENTRAL VS RADIOGRAFÍA DE TÓRAX PARA VERIFICAR LA ADECUADA COLOCACIÓN DEL CATÉTER VENOSO CENTRAL EN URGENCIAS.

\begin{tabular}{clccc} 
& & \multicolumn{2}{c}{ Radiografía de tórax } \\
\cline { 3 - 5 } & & Positivo & Neǵativo & Total \\
& & $\mathrm{N}(\%)$ & $\mathrm{N}(\%)$ & $\mathrm{N}(\%)$ \\
\hline \multirow{3}{*}{ OPVC } & Positivo & $123(99,1)$ & $3(75)$ & $126(98,4)$ \\
& Négativo & $1(0,8)$ & $1(25)$ & $2(1,5)$ \\
& Total & 124 & 4 & 128
\end{tabular}

IC 95\%

\begin{tabular}{lccc} 
& LI & LS \\
Se & $99,19 \%$ & 94,93 & 99,96 \\
Sp & $25 \%$ & 1,32 & 78,06 \\
VPP & $97,62 \%$ & 92,67 & 99,38 \\
VPN & $50 \%$ & 2,67 & 97,33 \\
\hline
\end{tabular}

* OPVC: Oscilación de la Presión Venosa Central; Se: Sensibilidad; Sp: Especificidad; VPP: Valor Predictivo Positivo; VPN: Valor Predictivo Negativo; IC 95\%: Intervalo de Confianza al 95\%; LI: Límite Inferior; LS: Límite Superior.

posibilidad inmediata de realizar radiografía de tórax, posterior a la colocación de los accesos vasculares centrales. Por tal motivo y, para evitar el retraso en el uso de la línea central para el tratamiento de los pacientes graves de las áreas de "Choque", se ha optado por practicar pruebas clínicas que orienten a determinar si el CVC está en ade- cuada posición, en tanto se espera corroborarlo con radiografía de tórax o ultrasonido $(4,5,18,19)$.

Chui y col. en el 2018, desestimaron el valor de la radiografía de tórax, cuando la compararon con el ultrasonido para corroborar la posición adecuada de los CVC y sus complicaciones, y concluyeron que la radio- 


\section{TABLA IV}

VALIDEZ DE MEDICIÓN EXTERNA VS RADIOGRAFÍA DE TÓRAX PARA VERIFICAR LA ADECUADA COLOCACIÓN DEL CATÉTER VENOSO CENTRAL EN URGENCIAS.

\begin{tabular}{|c|c|c|c|c|}
\hline & & Radio & tórax & \\
\hline & & $\begin{array}{c}\text { Positivo } \\
\text { N (\%) }\end{array}$ & $\begin{array}{c}\text { Negativo } \\
\text { N (\%) }\end{array}$ & $\begin{array}{l}\text { Total } \\
\text { N (\%) }\end{array}$ \\
\hline & Positivo & $124(100)$ & $4(100)$ & $128(100)$ \\
\hline ME & Negativo & 0 & 0 & 0 \\
\hline & Total & 124 & 4 & 128 \\
\hline & & & & \\
\hline & & LI & LS & \\
\hline $\mathrm{Se}$ & $100 \%$ & 96,25 & 99,93 & \\
\hline $\mathrm{Sp}$ & $0 \%$ & 2,35 & 60,42 & \\
\hline VPP & $96,88 \%$ & 91,71 & 98,99 & \\
\hline
\end{tabular}

" ME: Medición Externa; Se: Sensibilidad; Sp: Especificidad; VPP: Valor Predictivo Positivo; IC 95\%: Intervalo de Confianza al 95\%; LI: Límite Inferior; LS: Límite Superior.

TABLA V

VALIDEZ DE GASOMETRÍA VENOSA VS RADIOGRAFÍA DE TÓRAX PARA VERIFICAR LA ADECUADA COLOGACIÓN DEL CATÉTER VENOSO CENTRAL EN URGENCIAS.

\begin{tabular}{clccc} 
& & \multicolumn{2}{c}{ Radiografía de tórax } \\
\cline { 3 - 5 } & & Positivo & Neǵativo & Total \\
& & N $(\%)$ & N $(\%)$ & N (\%) \\
\hline \multirow{3}{*}{ GVC } & Positivo & $120(96,7)$ & $4(100)$ & $124(96,8)$ \\
& Negativo & $4(3,2)$ & 0 & $4(3,1)$ \\
& Total & 124 & 4 & 128
\end{tabular}

IC 95\%

\begin{tabular}{lccc} 
& LI & LS \\
Se & $96,77 \%$ & 91,45 & 98,96 \\
Sp & $0 \%$ & 2,35 & 60,42 \\
VPP & $96,77 \%$ & 91,45 & 98,96 \\
VPN & $0 \%$ & 2,35 & 60,42 \\
\hline "GVC: Gasometría Venosa Central; Se: Sensibilidad; Sp: Especificidad; VPP: Valor Predictivo Positivo; VPN: Valor \\
Predictivo Negativo; IC 95\%: Intervalo de Confianza al 95\%; LI: Límite Inferior; LS: Límite Superior.
\end{tabular}

grafía es más costosa e, incluso en muchas ocasiones, innecesaria (24); sin embargo, Roldan y col. en 2016, en una revisión sistemática, mantuvieron como estándar de oro a la radiografía de tórax, ya que es útil, no solo para detectar mala posición del CVC, sino para determinar malformaciones congénitas que pudieran ser la causa de la inadecuada posición de la punta de la línea central (8).
En nuestro estudio determinamos que las cinco pruebas clínicas fueron altamente sensibles, con un valor de Se por arriba del 90\%, con excepción de las arritmias, y con altos VPP, todas ellas por arriba del 90\%. En la literatura mundial, no existen muchos estudios que determinen la exactitud de pruebas clínicas, para corroborar la adecuada colocación del GVC, ante la ausencia inmediata de 


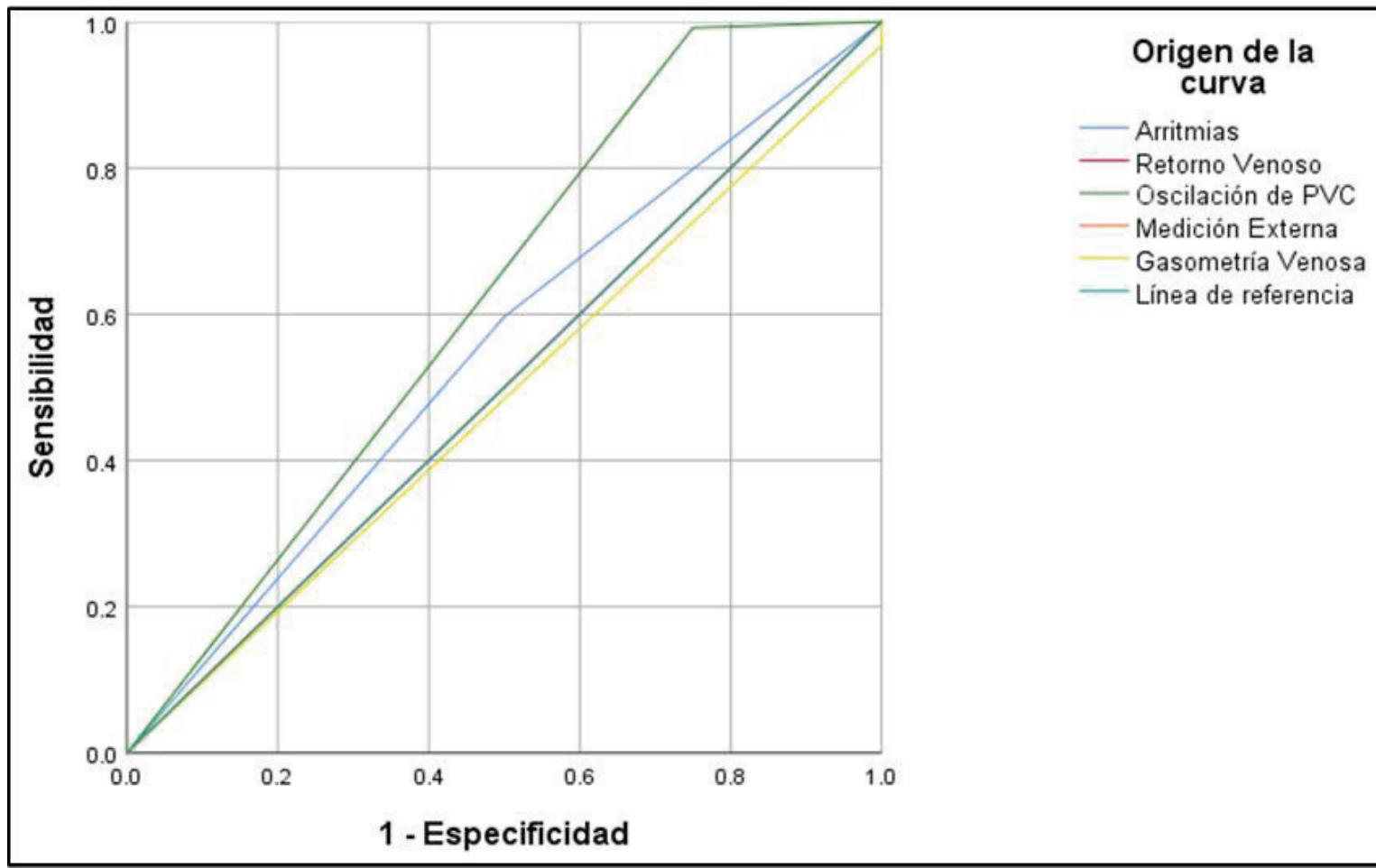

* Oscilación de PVC: Oscilación de Presión Venosa Central.

Fig. 3. Curvas de ROC de las pruebas clínicas para verificar la adecuada colocación del eatéter venoso central en Urgencias.

TABLA VI

RESULTADOS DE LA VALIDEZ DE LAS PRUEBAS CLÍNICAS PARA VERIFICAR LA ADECUADA COLOCACIÓN DEL CATÉTER VENOSO CENTRAL EN URGENCIAS.

\begin{tabular}{ccccccc}
\hline \multirow{2}{*}{$\begin{array}{c}\text { VARLABLES DE } \\
\text { RESULTADO DE PRUEBA }\end{array}$} & Se & Sp & \multicolumn{2}{c}{ VP } & \multicolumn{2}{c}{ IC 95\% } \\
\cline { 5 - 7 } & & & Positivo & Negativo & LI & LS \\
\hline Ar & $59,6 \%$ & $50 \%$ & $97,3 \%$ & $3,8 \%$ & 0,504 & 0,682 \\
RV & $100 \%$ & $0 \%$ & $96,8 \%$ & $0 \%$ & 0,962 & 0,999 \\
OPVC & $99,1 \%$ & $25 \%$ & $97,6 \%$ & $50 \%$ & 0,949 & 0,999 \\
ME & $100 \%$ & $0 \%$ & $96,8 \%$ & $0 \%$ & 0,962 & 0,999 \\
GVC & $96,7 \%$ & $0 \%$ & $96,7 \%$ & $0 \%$ & 0,914 & 0,989
\end{tabular}

* Ar: Arritmias; RV: Retorno Venoso; OPVC: Oscilación de Presión Venosa Central; ME: Medición Externa; GVC: Gasometría Venosa Central; Se: Sensibilidad; Sp: Especificidad; VP: Valores Predictivos; IC 95\%: Intervalo de Confianza al 95\%; LI: Límite Inferior; LS: Límite Superior. 
estudios de imaǵen. Martínez y col. en 2009, encontraron valores similares a los nuestros, con pruebas muy sensibles pero poco específicas, sin incluir a la GVC en su estudio (19).

El hecho de que las pruebas clínicas que proponemos tengan altos valores de Se y VPP, dan la certeza de que si al realizar estas, resultan positivas, es altamente probable que el CVC esté en el lugar correcto. En nuestra investigación, la prueba que obtuvo mejores valores de $\mathrm{Se}, \mathrm{Sp}, \mathrm{VP}$ y $\mathrm{ABC}$, es la OPVC, contrario a lo que Martínez concluyó en su estudio, puesto que las arritmias, fueron la prueba que obtuvo mejores valores de exactitud. Una limitante de nuestro estudio fue que la maniobra la realizó un solo médico, experto en la colocación de líneas centrales por una sola vía de abordaje (subclavia derecha), y pudiera tener mayor validez, si hubieran sido más los médicos que realizaron el procedimiento, por diferentes vías de acceso y con diferentes grados de experiencia. Para futuras investigaciones se podrían tomar en cuenta estas variables y así determinar si eso influye en la exactitud de las pruebas clínicas. Además, la validez de estas pruebas clínicas las estudiamos por separado, dando oportunidad para la realización de futuras investigaciones, que estudien la exactitud de las mismas en conjunto y determinar si mejoran sus valores. En países con economías emergentes, es de utilidad la práctica cotidiana de estas cinco pruebas clínicas, que representan instrumentos confiables para determinar la adecuada colocación del CVC. Hasta el momento no hay estudios que comparen la validez de estas pruebas con la del ultrasonido, por lo que se requiere la realización de más investigaciones para este fin.

El uso de estas pruebas puede optimizar el tiempo entre la colocación de la línea central y el inicio de su uso, lo que es de relevancia, para no retrasar el tratamiento en pacientes en estado crítico, que normalmente se atienden en los Servicios de Urgencias.
Por los altos valores de Se y VPP obtenidos en nuestro estudio, sugerimos llevar a cabo estas pruebas clínicas para corroborar la adecuada colocación del CVC, teniendo la seguridad de que si al realizarlas su resultado es positivo, se tendrá la certeza de que la línea central está correctamente colocada y se podrá iniciar la utilización de la misma, sin la necesidad de esperar a realizar ultrasonido o radiografía de tórax. Solo recomendaríamos que, al tener la sospecha de alguna complicación secundaria a la colocación del CVC, se realizara una prueba de imagen (radiografía de tórax o ultrasonido), para corroborar la existencia o no de la complicación sospechada.

Proponemos la realización de estudios futuros, con una casuística mayor, para determinar cuál prueba, o cuántas de estas cinco pruebas clínicas en conjunto, deben resultar positivas para tener una mayor seguridad para la no realización de la radiografía de tórax.

\section{REFERENCIAS}

1. European Centre for Disease Prevention and Control. Annual epidemiological report healtheare-associated infections acquired in intensive care units (internet). 2016-2017. Disponible en: https:// ecde.europa.eu/en/publicationsdata/ health care-associated infections acquired intensive eare units annual.

2. Ablordeppey EA, Drewry AM, Beyer AB, Theodoro DL, Fowler SA, Fuller BM, Carpenter CR. Diagnostic accuracy of central venous catheter confirmation by bedside ultrasound versus chest radiography in critically ill patients: a systematic review and meta-analysis. Crit Care Med 2017;45:715724.

3. Salimi F, Hekmatnia A, Shahabi J, Keshavarzian A, Reza MM, Davarpanah AH. Evaluation of routine postoperative chest roentgenogram for determination of the correct position of permanent central venous eatheters tip. J Res Med Sci 2015; 20:89-92. 
4. Wallace JA, Afonso E, Yu H, Birchard K, Isaacson A. Factors that predict increased catheter tip movement in left internal jugular vein implantable venous access ports upon standing. J Vase Access 2015:16(3):223-226.

5. Torres-Millán J, Torres-López M, Benjumea-Serna M. Location of the eentral venous eatheter tip in the right atrium: description in 2348 critical patients. Med Intensiva 2010;34: 595-599.

6. Dulce M, Steffen IG, Preuss A, Renz DM, Hamm B, Elgeti T. Topographic analysis and evaluation of anatomical landmarks for placement of central venous eatheters based on conventional chest X-ray and computed tomography. Br J Anaesth 2014;112:265271.

7. Marur VP, Manimala RS, Madhulika E. Can we predict the position of eentral venous catheter tip following cannulation of internal jugular vein? EC Anaesth 2015;2:126130.

8. Roldan, C, Paniagua, L. Central venous eatheter intravascular malpositioning: causes, prevention, diagnosis, and correction. West J Emerg Med 2015;16:658-664.

9. Oliveira L, Pilz L, Tognolo CM, Tognolo CM, Bischoff C, Becker KA, Oliveira GG, Neves PJ, Fachin CG, Agulham MA, Dias AIBS. Comparison between ultrasonography and X-ray as evaluation methods of central venous eatheter positioning and their complications in pediatrics. Pediatr Surg Int 2020;36:563-568.

10. Bodenham A, Babu S, Bennett J, Binks R, Fee P, Fox B, Johnston AJ, Klein AA, Langton JA, Melure H, Tighe SQM. Association of Anaesthesists of Great Britain and Ireland: safer vaseular aceess guidelines 2016. Anaesthesia 2016;71:573-585.

11. Raviraj R, Korula G, Subramani K, Shalinicynthia S. A simple method of electrocardiogram: Controlled central venous catheterization. Ann Card Anaesth 2011;1:154-155.

12. Meǵgiolaro M, Seatto A, Zorzi A, RomanPognuz E, Lauro A, Passarella C, Bonaccorso G. Confirmation of correct central venous eatheter position in the preoperative setting by echocardiographic "bubble- test". Minerva Anestesiol 2015;81(9):9891000 .

13. Gebhard R, Szmuk P, Pivalizza E, Melnikov V, Vogt C, Warter R. The accuracy of electrocardiogram-controlled central line placement. Anesth Analg 2007;104: 65-70.

14. Kumar A, Gupta K, Bhandari S, Singh R. Folding back of central venous catheter in the internal jugular vein: methods to diagnose it at the time of insertion? Indian J Anaesth 2013;67:104-105.

15. Blans MJ, Endeman H, Bosch FH. The use of ultrasound during and after central venous eatheter insertion versus conventional chest X-ray after insertion of a central venous eatheter. Neth J Med 2016;74:353357.

16. Weekes AJ, Keller SM, Efune B, Ghali S, Runyon M. Prospective comparison of ultrasound and CXR for confirmation of central vascular catheter placement. Emerg Med J 2016;33:176-180.

17. Raman D, Sharma M, Moghekar A, Wang $X$, Hatipoğlu U. Utilization of thoracic ultrasound for confirmation of central venous eatheter placement and exclusion of pneumothorax: a novel technique in realtime application. J Intensive Care Med 2019;34:594-598.

18. Krishnan AK, Menon P, Gireesh Kumar KP, Sreekrishnan TP, Garg M, Kumar SV. Electrocardiogram-guided technique: an alternative method for confirming central venous eatheter tip placement. J Emerg Trauma Shock 2018;11:276-281.

19. Martínez FF, Márquez GH, Márquez FH, Rodríguez RE, Guerrero AM. Validez de las pruebas clínicas para determinar posición del catéter venoso central. Rev Med Inst Mex Seg Soc 2009;47:665-668.

20. Brenner B, Gibson F, Bodenham A. Misplaced central venous catheters: applied anatomy and practical management. $\mathrm{Br} \mathrm{J}$ Anaesth 2013;110:333346.

21. Smith RN, Nolan JP. Central venous eatheters. BMJ 2013;347:1-11.

22. Byrne AL, Bennett M, Chatterji R, Symons R, Pace NL, Thomas PS. Peripheral venous and arterial blood gas analysis in adults: are they comparable? A systematic review and meta-analysis. Respirology 2014;19:168-175. 
23. Mallat J, Lazkani A, Lemyze M, Pepy F, Meddour M, Gasan G. Repeatability of blood gas parameters, $\mathrm{PCO}_{2}$ gap, and $\mathrm{PCO}_{2}$ gap to arterial-to-venous oxyǵen content difference in critically ill adult patients. Medicine (Baltimore) 2015;94:1-6.

24. Chui J, Saeed R, Jakobowski L, Wang W, Eldeyasty B, Zhu F, Fochesato LA, Lavi R, Bainbridge D. Is routine chest X-ray after ultrasound-guided central venous catheter insertion choosing wisely? A populationbased retrospective study of 6,875 patients. Chest 2018;154(1):148-156.

25. Chebl RB, Kiblawi S, Khuri CE, Hajj NE, Bachir R, Aoun R, Daghher GA. Use of contrast-enhanced ultrasound for confirmation of central venous catheter placement. J Ultrasound Med 2017;36:2503-2510. 